\title{
INTEGRATED LT CODING, BIT INTERLEAVED DIFFERENTIAL SPACE TIME CODING AND SPHERE PACKING MODULATION FOR THE WIRELESS INTERNET
}

\author{
N. Wu, T.D. Nguyen, C.Y. Wei, L.L. Yang and L. Hanzo \\ School of ECS, University of Southampton, SO17 1BJ, UK. \\ http://www-mobile.ecs.soton.ac.uk
}

\begin{abstract}
In this treatise, a jointly optimized Bit Interleaved Coded Modulation using Iterative Decoding (BICM-ID) and Luby Transform (LT) coding scheme was proposed for the sake of attaining the best possible performance, when communicating over the wireless Internet. This scheme is particularly attractive when handling packetized wireless Internet data traffic received over wireless channels inflicting both statistically multiplexing induced packet erasure and packet corruption imposed by the channel without retransmitting the corrupted packets. A LLR-based estimation packet reliability algorithm was proposed to assist the LT decoder in recovering all the transmitted packets. Furthermore, the bit-to-sphere-packingsymbol mapping scheme was optimized for achieving error free LT decoding at the lowest possible $E_{b} / N_{0}$ value rather than for approaching the channel's capacity. These two design philosophies require different mapping schemes. Finally, Differential Space Time Coding (DSTBC) was employed for combatting the effects of fading and provided spatial diversity at the same time. The LT code's packet length was also carefully chosen to strike a good balance between the affordable complexity and the achievable performance.
\end{abstract}

\section{INTRODUCTION}

The evolution of the wireless Internet is driven by the simultaneous need for high data rates and reliable transmission, for supporting attractive services such as online banking, audio and video download, Voice-Over-IP (VOIP) telephone, IP-TV etc. In the Internet, data is transmitted in form of packets. Each packet is provided with a header, amongst others containing the identifier of both the source and destination. These packets are routed through the network from the transmitter to the receiver. Owing to statistical multiplexing induced packet dropping and buffer overflows at the intermediate router nodes, some packets may get lost and hence never reach their destination. Other packets may have to be erased, if the receiver's channel decoder fail to recover all channel errors and this was detected by Cyclic Redundancy Checksum (CRC). This multi-faceted packet loss phenomenon may be mimicked by the Binary Erasure Channel (BEC) model proposed in [1].

To ensure reliable data transmission over the wireless Internet, various protocols may be used to compensate for the erased packets. For example, the ubiquitous TCP/IP ensures infinitesimally low packet loss rate by retransmitting packets, whose reception has not been acknowledged by the receivers within a given window. However, packet acknowledgement (ACK) based mechanism may generate an excessive transmission overhead, for example, when the transmitter sends numerous unnecessary copies of the same packet owing to an excessive acknowledgement window encountered in

The financial support of the EPSRC, UK and that of the EU under the auspices of the OPTIMIX project are gratefully acknowledged. case of long propagation distances. Similarly, in broadcasting scenario, the sender will have to transmit a high number of redundant packets, even if all but one receivers have already received the retransmitted packets.

To address these issues, Luby Transform (LT) codes [2] [3] were proposed by adopting a philosophy reminiscent of Fountain codes [4], which require no acknowledgement and benefit from fast encoding and decoding algorithms. More explicitly, instead of using an explicit packet ACK mechanism to request the retransmission of redundant packets, LT codes generate controllable amount of redundant packets. The redundant LT encoded packets will assist the receiver in retrieving all the transmitted data packets potentially erased by the BEC.

The employment of multiple antennas at both the transmitter and receiver of a wireless communication system offers potentially high diversity gains and high data rates. However, because of the typically asymmetric throughput requirement of the Up-Link (UL) and Down-Link (DL), the design of the high-throughput DL is often more challenging, especially in high-rate DL file-transfer applications routinely encounter in wireless Internet applications.

Bit Interleaved Space Time Coded Modulation using Iterative Decoding (BI-STCM-ID), combines Bit-Interleaved Coded Modulation (BICM) [5] employing both iterative demapping and decoding [6] with the principle of Space-Time Block Codes (STBC) [7] [8]. The amalgamated scheme becomes capable of capturing both space as well as time diversity and hence achieves a high performance under a wide variety of fading conditions. Differential Space-Time Block codes (DSTBC) [9] are capable of achieving the maximum attainable diversity gain in DL transmission without the overhead of transmitting channel-sounding pilot symbols for the sake of coherent detection. As an attractive design alternative, in this contribution we explorer the potential of differentially detected BI-DST-CM-ID, which requires no channel-sounding.

In [10], the authors investigated the joint benefit of Forward Error Correction (FEC) codes and LT codes where the source transmitted a limited number of redundant packets using LT encoding, which assisted the receiver in recovering all the original data packets, even if the FEC code failed to remove all the errors in some of the packets, which hence had to be erased for the sake of avoiding avalanche-like LT-decoder induced error propagation. As in most of the schemes found in the literature in addition to the desired data, the 16-bit CRC, which instructed the LT decoder to erase the erroneous packets, also has to be transmitted. However, the CRC overhead may become potentially high when the length of the LT packet is relatively low.

In this paper, we proposed an integrated physical layer design, amalgamating the above-mentioned BI-DST-CM-ID and LT codes for employment in a wireless Internet scenario. The novelty and rationale of this paper can be summarized as follows:

- Packet-reliability estimation to avoid the CRC-overhead. In order To remove the CRC overhead, a Log Likelihood Ratio 


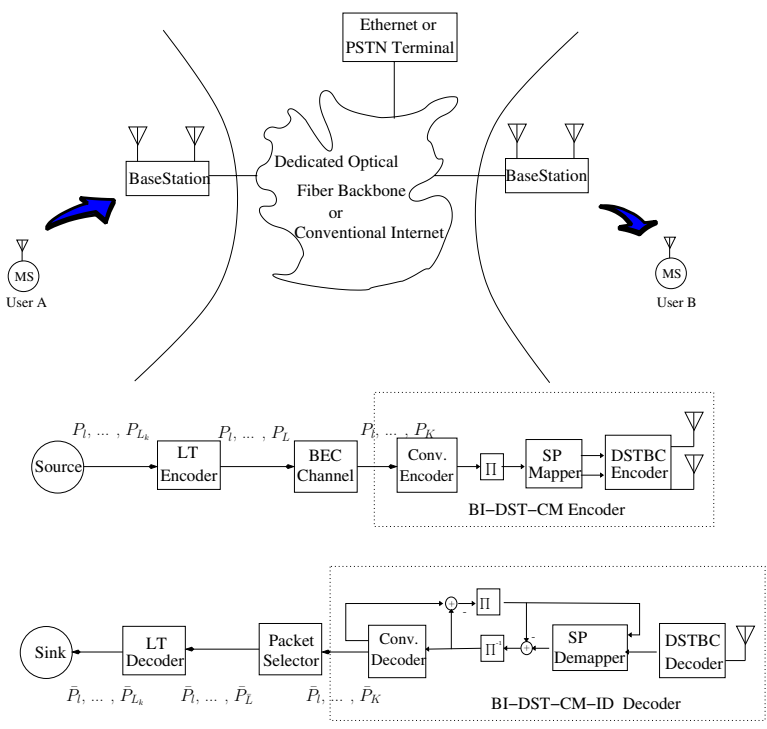

Fig. 1. Real world scenario and the corresponding transceiver schematic of the proposed LT and BI-DST-CM integrated scheme.

(LLR) based estimation algorithm is proposed for assisting the LT decoder in removing the erroneous packets caused by the channel impairment when the channel decoder failed to remove them. Thus, the integrated system becomes capable of avoiding the potentially avalanche-like LT decoding induced error propagation.

- Mapping design. It is widely recognized that the design of modulation constellation mapping pre-determines the achievable performance of iteratively decoded schemes [11] [12]. We will demonstrate that with the aid of Extrinsic Information Transfer (EXIT) charts [13] [14], we are capable of designing a beneficial mapping arrangement suitable for LT encoded schemes, additionally employing Sphere Packing (SP) modulation [15] for achieving the highest possible iteration gain.

- Differential Space-Time Block Coding (DSTBC) is integrated with iterative decoding aided BICM [15] in order to minimize the effects of fading-induced burst errors as well as to maximize the attainable spatial and time diversity order. The improved robustness of the "inner" BI-DST-CM-ID scheme is expected to reduce the number of redundant data packets required for recovering all the original data packets.

We commence our detailed discourse by providing a description of the proposed system in Section II as well as various system parameter designs. While our simulation results are discussed in Section III. Finally, we conclude our discourse in Section IV.

\section{JOINT DESIGN OF THE LT AND BI-DST-CM-ID SCHEME}

\section{II-A. System Model}

Let us assume that Mobile Station (MS) A wants to send a data file to MS B. We assume that the Base Stations (BS) are connected either by a dedicated optical fiber backbone or via the conventional Internet, as seen in Figure 1. In this treatise, we consider the DL, hence and assume that no impairments are imposed by the UL. There are two main sources of packet loss or corruption. Firstly, the conventional Internet may drop packets due to statistically multiplexing induced congestion. Secondly, the wireless DL of Figure 1 may impose fading and AWGN induced packet corruption. In order to highlight the system's architecture more explicitly, the schematic of the corresponding transmission scenario is further depicted at the bottom of Figure 1. More explicitly, the conventional Internet is modeled by the BEC channel, which drops packets randomly. By contrast, the wireless link impose the non-dispersive Rayleigh fading and AWGN.

Firstly, the data file to be transmitted is partitioned into $L_{k}$ number of fixed-length packets $P_{1}, \ldots, P_{L_{k}}$. Each packet contains $(n \times m)$ bits, where $n$ denotes the number of Sphere Packing (SP) symbols transmitted within a single LT-encoded packet, while $m$ is the number of information bits per SP symbol. For the sake of reliably recovering the entire original file, the LT encoder seen in Figure 1 generates $L=L_{k}+L_{e}$ number of packets from the incoming $L_{k}$ packets, where $L_{e}$ is the number of redundant packets that has to be as low as possible. The so-called improved robust degree distribution of LT codes [10] was used for the sake of achieving as low packet overhead, i.e. $L_{e}$ as possible ${ }^{1}$ Then, the LT-protected packets are transmitted over the Internet, which is, again modeled as a BEC having an erasure probability of $P_{e}$. The remaining $K=L \times\left(1-P_{e}\right)$ number of packets successfully reach BS B of Figure 1. The BI-DST-CM encoder of BS B is equipped with multiple antennas, as seen in Figure 1, where a number of LT-encoded packets may be concatenated to form a transmission frame. Then, after interleaving which is implicit in the BI-DST-CM encoder, each encoded frame is transmitted using SP modulation. The modulated symbols are differentially encoded using DSTBC and transmitted. The detailed description of the BIDST-CM encoder can be found in [15].

The wireless communication medium is modeled as a correlated non-dispersive Rayleigh fading channel. The rate of fading is governed by the normalized Doppler frequency given by $f_{d}$. We assume that the spatial separation between the twin transmit antennas of the BS is on the order of 5-10 times the wavelength of the carrier frequency used, in order to ensure that the fading experienced by all diversity channels becomes independent.

At the receiver side, the BI-DST-CM-ID decoder outputs the LLRs for each single bit and generates the LLR-based packets $\bar{P}_{1}, \ldots, \bar{P}_{K}$ of soft-values. Since the LT decoder only requires $\bar{L}$ number of packets for recovering the data file, the packet selector of Figure 1 chooses appropriately optimized $\bar{L}$ packets, which contains the lowest number of errors from the BI-DST-CM-ID decoder based on the proposed LLR-based packet reliability estimation algorithm, which will be outlined immediately in Section II-B. Finally, the LT decoder [10] of Figure 1, recovers the data file using the $\bar{L}$ number of unerased packets, which are likely to be error-free and hence avoids the otherwise inevitable avalanche-like LT decoding error propagation.

\section{II-B. LT- Packet Selection Based on LLR Estimation}

As alluded to above, the packet selector of Figure 1 need to choose the most "reliable" $\bar{L}$ packets from the entire set of $K$ number of packets. However, if the number of highly reliable (estimated error-free) packets is insufficient for successful LT decoding, a number of less reliable BI-DST-CM-decoded packets may also be passed to the LT decoder. In other words, we may allow the packet selector to drop $S=K-\bar{L}, S<<\bar{L}$ number of potentially less reliable packets. More explicitly, we

\footnotetext{
${ }^{1}$ The LT code's degree distribution determines the number of original input packets, which are used for generating the LT-encoded packets. For example, a degree one packet is directly mapped without any modulo two operation to an LT packet, while degree- $N$ packets depend on $N$ input packets.
} 


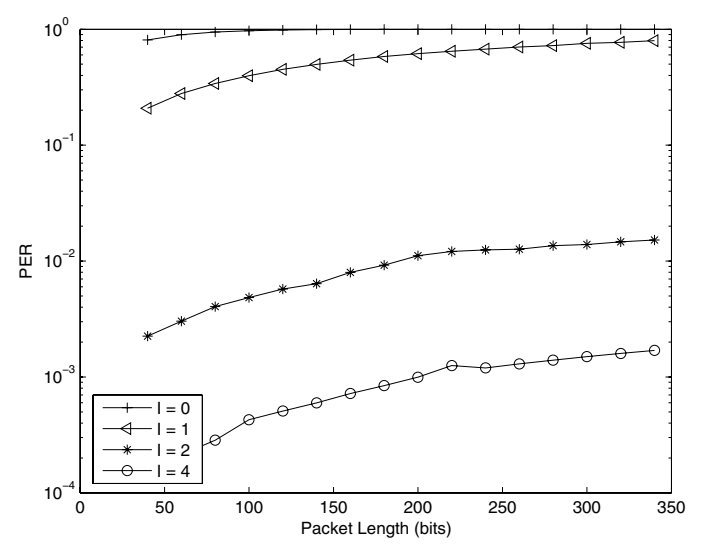

Fig. 2. PER performance for different LT packet lengths using $I=0,1,2$, and 4 BI-DST-CM-ID decoder iterations at $8 d B$, when recorded before the packet selector seen in Figure 1.

need a sufficiently high number of sufficiently reliable packets for completing the LT decoding.

Again, our goal is to select sufficiently high number of sufficiently reliable packets for the LT decoder to achieve its best possible performance. The philosophy of the of LLR-based packet reliable estimation algorithm is to define a certain threshold, $t>0$, and if any bit's LLR value is within the interval $[-t, t]$, then we consider the bit to be unreliable. By contrast, if the LLR value is outside this unreliable region, this bit is assumed to be reliably, i.e. to be correct. Based on this method, we consider the packets that contained the most errors to be erased. The key to the success of this algorithm is to choose a suitable reliability threshold for various decoding scenarios, such as having a different affordable number of iterations, $E_{b} / N_{0}$ and other channel conditions.

Recall that we only need have to erase $S$ number of less reliable packets and forwarding the rest of high reliable packets to the LT decoder. Therefore, we contrived a simple algorithm to search for the most beneficial threshold value for each specific scenarios, which is outlined below:

- Initially, we set $t=0.1 \times \max (L L R)$, since this value allow us to maintain the number of search steps performed low.

- if the number of estimated error packets smaller than $S$, then increase $t$ gradually, until this number become equal to $S$.

- if the number of estimated error packets larger than $S$, then decrease $t$ gradually, until this number become equal to $S$.

\section{II-C. Packet Length}

Since the LT decoder uses a packet based algorithm, the system's Packet Error Rate (PER) recorded at the output of the BI-DSTCM-ID decoder of Figure 1 is a more important parameter than its Bit Error Rate (BER). More explicitly, recall that the inner BIDST-CM encoding consisted of the convolutional code specified in Table I, bit-based interleaver and a DSTBC assisted SP modulator. In this section, we will demonstrate the associated design tradeoffs between the LT decoder's complexity and its PER performance.

Figure 2 portrays the BI-DST-CM-ID inner decoder's PER performance, when employing different number of iterations at $8 d B$, using different LT-encoded Packet Lengths (PL). Observe that for a certain number of iterations, the PER was increased when the packet length was increased. Since the errors were more-or-less uniformly distributed after the bit-based interleaver, we expect to

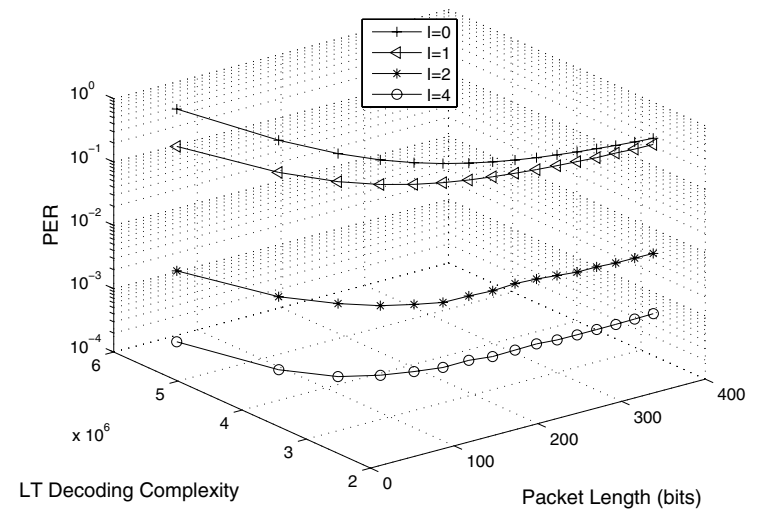

Fig. 3. PER, LT packet size and LT decoding complexity tradeoff, using 0,4 and 8 iterations (iter) for BI-DST-CM-ID decoder at $8 d B$, when measured before the packet selector as seen in Figure 1.

have an increased PER, when more information bits are packed into a single LT packet. The number of BI-DST-CM-ID iterations quite dramatically influenced the achievable PER. Naturally a higher number of iterations resulted in a lower PER. A better PER performance was attained for lower LT packet lengths. However, a lower LT packet size increases the LT's decoding complexity, as shown in Figure 3, expressed in terms of the number of search in LT's generation matrix, as it will be detailed below.

Figure 3 explicitly shows the tradeoffs between the PER, LT packet size and LT decoding complexity when the inner BIDST-CM-ID decoder employs $I$ number of iterations at $8 d B$. More explicitly, the LT decoder's complexity is constituted by two contributors. One is the number of bit-based modulo two additions, which is independent of the packet size. The second contributor is the total number of search operations to be carried out in the LT generator matrix to extract the right packet to perform modulo-two operation. For example, a degree $n$ packet required $n$ number of search. Since the complexity of the above-mentioned modulo-two operations is independent of the packet size, in this paper, the LT decoder's complexity is quantified in terms of the number of search operations in LT code's generator matrix, which is given by:

$$
C_{l t}=(P L+N) \times \bar{L},
$$

where $N$ is the highest degree of the LT encoded packets. It also can be understood as the maximum weight of rows of the LT generator matrix. Recall $\bar{L}$ is the number of packets feed into LT's decoder as seen in Figure 1. As seen in Figure 3, the appropriate choice of the LT code's packet size provides an attractive balance between the achievable PER and the LT decoder's complexity, when jointly considering the performance trends observed in Figures 2 and 3, $P L=120$ was chosen for our proposed scheme.

\section{II-D. Mapping Design}

It is widely recognized known that the specific mapping of bits to transmitted symbols has a high impact on the system's performance in particular in iteratively decoded scenarios [11]. This is exemplified in [16] in the context of BICM-ID and Turbo Trellis Coded Modulation (TTCM). The exchange of extrinsic information between the convolutional decoder and the DSTBC assisted SP demapper can be visualized using Extrinsic Information Transfer (EXIT) charts [14], as seen in Figure 4. It was shown 


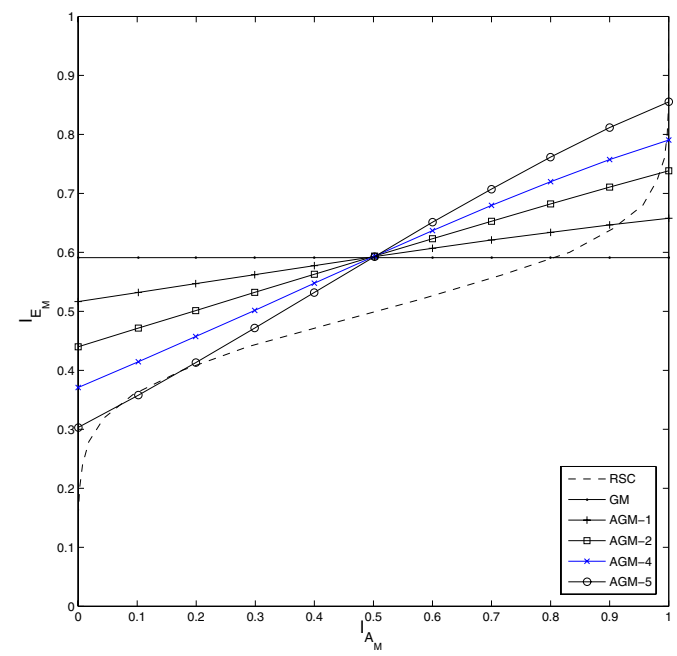

Fig. 4. DSTBC assisted Sphere Packing (SP) demapper [15] EXIT characteristics for Gray Mapping (GM) and for four different bits to SP symbol Anti-Gray-Mapping (AGM) schemes at $7 d B$, where each SP symbol contains 4 bits.

in [14] that the EXIT charts are capable of reliably predicting the achievable BER performance. However, for our proposed scheme it is the inner code's PER rather than its BER which determines the "outer" LT decoder's attainable performance. Nevertheless, since the interleaver distributes the errors more-or-less uniformly, it is plausible that a system having a lower BER may be expected to maintain a lower PER.

The conventional design goal of iterative systems is to minimize BER, which requires that the extrinsic transfer curves intersect at the $\left(I_{A_{M}}, I_{E_{M}}\right)=(1.0,1.0)$ point [14], where $I_{A_{m}}$ and $I_{E_{m}}$ denotes the mutual information of for inner BI-DST-CM system's input and output respectively. If this condition is satisfied, then a so-called open convergence tunnel [14] appears in the EXIT chart. Even if there is no open tunnel in the EXIT chart leading to the $(1.0,1.0)$ point, but the two EXIT curves intersect at a point close to $I_{E_{M}}=1.0$, then a sufficiently low BER may still be achievable.

Figure 4 shows the EXIT chart of our half rate Recursive Systemic Convolutional code $\operatorname{RSC}(2,1,5)$ and that of five different bit-to-SP-symbol mapping schemes, where classic Gray Mapping (GM) is outperformed by all the four Anti-GM (AGM) schemes, which were explicitly defined in [15]. The AGM-5 scheme appears to be the best mapping scheme having a crossover withe the EXIT curve of the $\operatorname{RSC}(2,1,5)$ code at $(1,0.89)$. However, for our proposed scheme, the LT decoder benefiting from the previously proposed packet selector of Figure 1, is capable of recovering the data file even if there were a considerable number of residual errors, following the action of the BI-DST-CM-ID decoder. Therefore, the aim of the mapping design is that of creating an open convergence tunnel in the EXIT chart at as low as SNR as possible, while reaching a sufficiently high $I_{E_{M}}$ point at the same time. We will show in Section III that the AGM-4, which is also characterized in Figure 4 will be the most beneficial for employment by the LT detector in order to recover all the original data packets.

\section{SYSTEM PERFORMANCE}

Consider the situation, where a data file containing $1.2 \times 10^{6}$ bits has to be transmitted over the wireless Internet. Based on our discussions in Section 2, the LT packet size was set to
Table I. System Parameters

\begin{tabular}{|l|r|}
\hline LT Packet size $(n \cdot m)$ & 120 bits \\
\hline LT Distribution & Improved robust distribution [10] \\
\hline Number of information packets & $\left(L_{k}\right)=10,000$ \\
\hline Number of redundant packets & 3,000 \\
\hline Erasure probability of BEC & 0.1 \\
\hline $\begin{array}{l}\text { RSC with } \\
\text { octal generator polynomial }\end{array}$ & $\left(G_{r}, G\right)=(35,23)_{8}$ \\
\hline Interleaver length & $10^{6}$ bits \\
\hline Modulation & BI-DST-CM-SP, L=16 \\
\hline Mapping & AGM-4 [15] \\
\hline Normalized Doppler frequency & $f_{d}=f_{D} \cdot T=0.01$ \\
\hline
\end{tabular}

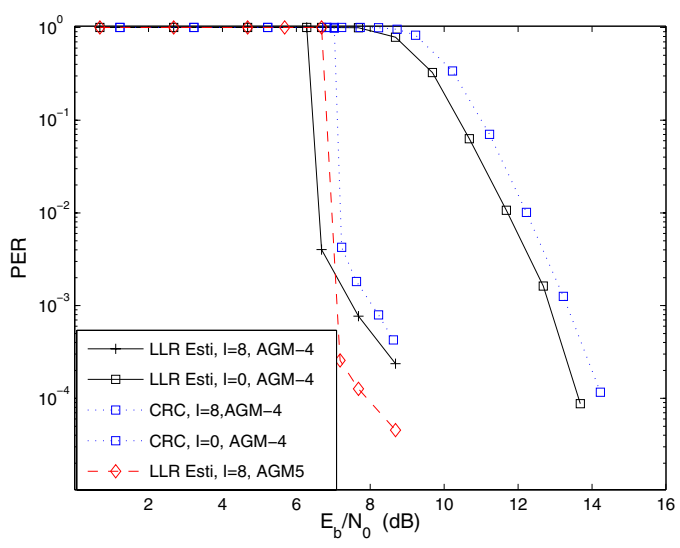

Fig. 5. PER of inner BI-DST-CM-ID code employing AGM-4 and AGM-5, for both the LLR based estimation and for the CRC algorithm, using $I=8$ iterations and non-iterative decoding.

$n \cdot m=120$ bits in order to achieve a balance between the achievable performance and the complexity imposed. Thus, the data file was splitted into $L_{k}=10,000$ packets and the LT encoder was configured to generate $30 \%$ redundant packets in order to counteract the packet loss events imposed by the BEC. Thus the result $L=13,000$ LT-encoded packets was transmitted over the conventional Internet. The erasure probability of the BEC was set to $P_{e}=0.1$, which implies that $L \times 0.1=1,300$ packets was erased by the conventional Internet section of the transmission. When erasure events happened, the BS in Figure 1 of user B receive nothing, Therefore BS has the knowledge of which packet is erased. As a result, only $K=13,000-1,300=11,700$ packets were transmitted over the wireless link. Then, a group of LT encoded packets were convolutionally encoded by the half rate $\operatorname{RSC}(2,1,5)$ code and were transmitted using two antennas. The overall code rate of this system was $r=10,000 /(13,000 \times(1-0.1) \times 2) \approx 0.427$. We found that at $P_{e}=0.1$ the LT decoder seen in Figure 1 required 11,000 LT packets to recover all the packets of the original data file. Although it is worth noting that the number of required packets depends not only on $P_{e}$ but also on degree-distribution used, which means we have the freedom of erase $S=11,700-11,000=700$ packets based on LLR estimation. The detailed system parameters are listed in Table I.

In order to characterize the proposed system in more detail, Figure 5 shows the "inner" BI-DST-CM's PER versus the $E_{b} / N_{0}$ using both non-iterative detection and $I=8$ iterations, when employing both CRC and LLR estimation based packet reliability estimation algorithms. The proposed LLR based packet reliability estimation based scheme used 120 bits per LT packet, as listed in Table I. On the other hand, the CRC based design alternative has 


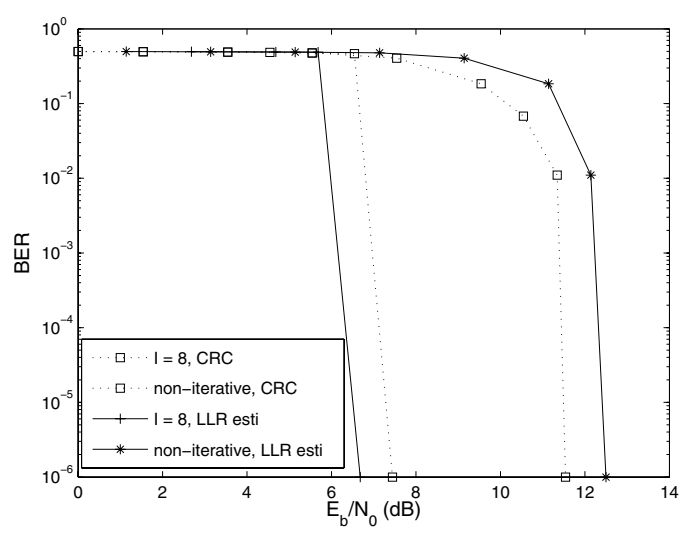

Fig. 6. BER comparison for the proposed scheme using both the LLR based packet reliability estimation and the CRC-based algorithms.

to transmit 16 additional bits for each LT encoded packet, which resulted in an extra $13.3 \%$ redundancy. Then the whole 136 bits are encoded by the BI-DST-CM encoder instead of 120 bits. At the receiver side, CRC was used to check the packet integrity instead of the "Packet selector". After the half rate RSC encoding, the resultant packet are now 32 bits longer and hence are more likely to become error-infected during their flight over the wireless channel, which inherently increases the PER, as quantified in Figure 5. The resultant potentially degraded inner code PER, which became detrimental for the LT decoder. Figure 5 explicitly show that our proposed LLR-based packet reliability estimation scheme exhibits a $0.5 d B$ advantage over the CRC based scheme for both non-iterative and 8 iterations.

Since the CRC based scheme guarantees the erroneous packet will be erased, once the number of erroneous received packets become less than $S$ as a benefit of increased $E_{b} / N_{0}$, the LT decoder becomes capable of perfectly recovering the data file. We found in our investigations not reported here owing to space economy that the $E_{b} / N_{0}$ required is $7.2 d B$ for $I=8$ iterations and $11.7 \mathrm{~dB}$ for non-iterative.

Our proposed LLR based packet reliability estimation algorithm is also capable of selecting packets, when a certain $E_{b} / N_{0}$ value is reached. The corresponding $E_{b} / N_{0}$ required for infinitesimally low error-free packet classification is $6.7 d B$ for $I=8$ iterations and $12.2 d B$ for non-iterative, when AGM-4 is employed. The reason for the LLR-based packet reliability estimation scheme's better PER performance is that it dispenses with the CRC-based overhead, which requires a commensurate shifting of the $\mathrm{CRC}$ related move to the right.

We also show the PER for AGM-5 using $I=8$ decoding iterations in Figure 5. As predicted in Figure 4, compared with AGM-4, its "turbo cliff" is delayed about $0.3 d B$, while reaching lower error floor at the same time. However, the lower error floor does not benefit the later stage LT decode, due to AGM-4's error floor has been low enough for LT decoder to recover all the transmitted packets.

Figure 6 show the attainable BER performance for both the LLR-based packet reliability estimation and CRC-based schemes. Observe that the proposed LLR based packet reliability estimation scheme outperforms its CRC counterpart by about $0.5 d B$ when using $I=8$ iterations. By contrast, a $0.5 d B E_{b} / N_{0}$ performance loss is recorded for the system employing non-iterative detection.
This observation corresponds to the $E_{b} / N_{0}$ requirement for the LT decoder perfectly recovering all the tranission packets in Figure 5.

\section{CONCLUSION}

An integrated LT code and BI-DST-CM design using iterative decoding was proposed for transmission over AWGN contaminated and non-dispersive Rayleigh faded wireless Internet channels, when the conventional Ethernet-based Internet was modeled by the BEC. The joint design of the DSTBC assisted BICM-ID and LT code's parameters and the associated bit-to-SP-symbol mapping scheme allowed us to improve the attainable BER performance of packetized data transmission. A LLR based packet reliability estimation algorithm was also proposed for removing the conventional CRC scheme's transmission overhead, while achieving a better performance at the same time. The LT code's packet length was also considered for the sake of striking a good balance between the implementation complexity imposed and the performance attained. Furthermore, instead of aiming for achieving the lowest possible BER for the inner code of Figure 1, the bit-to-SP-symbol mapping scheme was chosen for ensuring that the LT decoder become capable of near-error-free packet recovering at as low SNR as possible. The proposed scheme is particularly attractive for employment in a wireless Internet scenario, where the LT code for recovering erased packets and the BI-DST-CM-SP scheme is used for minimizing the error events inflicted by both fading and the AWGN. Our future research will focus on facilitating soft information exchange between the BI-DST-CM and LT component codes in order to further improve the system's performance.

\section{REFERENCES}

[1] P. Elias, "Coding for two noisy channels," in Proceedings of 3rd London Symp. on Information Theory, Septerber 1955.

[2] M. Luby, "LT codes," The 43rd Annual IEEE Symposium on Foundations of Computer Science, pp. 271-280, Nov. 2002.

[3] D. MacKay, "Fountain codes," IEE Proceedings Communications, vol. 152, pp. 1062-1068, Dec. 2005.

[4] D. MacKay, Information Theory, Inference and Learning Algorithms. Cambridge University Press, October 2003.

[5] G. Caire, G. Taricco, and E. Biglieri, "Bit-interleaved coded modulation," IEEE Transactions on Information Theory, vol. 44, no. 3, pp. 927-946, 1998.

[6] X. Li and J. Ritcey, "Bit-interleaved coded modulation with iterative decoding using soft feedback," Electronics Letters, vol. 34, no. 10, pp. 942-943, 1998.

[7] S. M. Alamouti, "A simple transmit diversity technique for wireless communications," IEEE Journal on Selected Areas in Communications, vol. 16, no. 8, p. $1451,1998$.

[8] V. Tarokh, H. Jafarkhani, and A. R. Calderbank, "Space-time block coding for wireless communications: performance results," IEEE Journal on Selected Areas in Communications, vol. 17, no. 3, p. 451, 1999.

[9] V. Tarokh and H. Jafarkhani, "A differential detection scheme for transmit diversity," IEEE Journal on Selected Areas in Communications, vol. 18, no. 7, pp. $1169-1174,2000$.

[10] R. Tee, T. Nguyen, L. Yang, and L. Hanzo, "Serially concatenated luby transform coding and bit-interleaved coded modulation using iteratlive decoding for the wireless internet," IEEE Vehicular Technology Conference, Melbourne Spring, vol. 1, pp. 22-26, August 2006.

[11] A. Chindapol and J. Ritcey, "Design, analysis, and performance evaluation for BICM-ID with square QAM constellations in rayleigh fading channels," IEEE Journal on Selected Areas in Communications, vol. 19, no. 5, pp. 944-957, 2001.

[12] Y. Li and X.-G. Xia, "Constellation mapping for space-time matrix modulation with iterative demodulation/decoding," IEEE Transactions on Communications, vol. 53, no. 5, pp. 764-768, 2005.

[13] S. ten Brink, J. Speidel, and R.-H. Yan;, "Iterative demapping and decoding for multilevel modulation," IEEE Global Telecommunications Conference, GLOBECOM, vol. 1, pp. 579 - 584, November 1998.

[14] S. ten Brink, "Designing iterative decoding schemes with the extrinsic information transfer chart," AEÜ International Journal of Electronics and Communicatioons, vol. 54, pp. 389-398, 2000.

[15] O. Alamri, N. Wu, and L. Hanzo, "A differential turbo detection aided sphere packing modulated space-time coding scheme," IEEE Vehicular Technology Conference, vol. 5, pp. 2474 - 2478, Spring 2006.

[16] L. Hanzo, T. H. Liew, and Y. B. L., Turbo Coding, Turbo Equalisation, and Space-Time Coding: For Transmission over Fading Channels. John Wiley \& Sons Inc, 2002. 